\title{
Occurrence of higher thiadiamondoids and diamondoidthiols in a Lower Cambrian oil from ZS1C well in Tazhong Uplift, Tarim Basin
}

\author{
ANLIA MA ${ }^{1}$, ZHIJUN JiN ${ }^{1}$, CUISHAN ZHU ${ }^{2}$, Yi GU ${ }^{1}$
}

1.Petroleum Exploration \& Production Research Institute, SINOPEC, No. 31 Xueyuan Road, Beijing, 100083; maal.syky@sinopec.com

2. Yangtze University, Wuhan, Hubei, 430100

A series of higher thiadiamondoids and diamondoidthiols, including thiatetramantanes, tetramantanethiols, thiapentamantes and pentamantanethiols were discovered in ZS1C well condensate in deep reservoir from Lower Cambrian Xiaoerbulake Formation in Tazhong Uplift, Tarim Basin, NW China. The ZS1C oil was associated with abundant lower thiadiamondoids including thiaamantanes, thiadiamantanes, thiatriamantanes, with concentration of $8578 \mu \mathrm{g} / \mathrm{g} \times$ oil. Higher diamondoids, such as tetramantanes, pentamantanes, hexamantanes and cyclohexmantane were also detected in the saturated fractions of ZS1C condensates. The oil is rich in lower diamondoids, with concentration of $87834 \mu \mathrm{g} / \mathrm{g} \times$ oil. The origin of higher thiadiamondoids and diamondoidthiols is similar to that of lower thiadiamondoids and diamondoidthiols. During TSR, the cage of higher diamondoids is interpreted to open because of sulfur radicals forming open-cage higher diamondoid-like thiols, followed by cyclization that leads to the formation of higher thiadiamondoids.

The concentration of diamondoids and thiadiamondoids of ZS1C oil is the highest in the marine oils in Tarim Basin, indicating ZS1C oil is a residual oil suffered serious TSR. This is also support by the sulfur isotope of whole oil and thiadiamondoids (Cai et al.,2016).

With exploration moving to the deep strata, Middle Cambrian in Tabei and Tazhong Uplifts with depths greater than $8000 \mathrm{~m}$ and temperature greater than $170^{\circ} \mathrm{C}$, developed a succession sediments of anhydrite and salt beds. Reactive sulfate for TSR was likely derived from the dissolution of anhydrite of Middle Cambrian. Although the degree of TSR in the Ordovician reservoirs is low, greater degree of TSR might occur in the deep Cambrian at depths $>8000 \mathrm{~m}$. 